\title{
Correlation between Gross Motor Function Classification System and Communication Function Classification System in Children with Cerebral Palsy
}

\author{
Vindy Margaretha Miguna, ${ }^{1}$ Marietta Shanti Prananta, ${ }^{2}$ Anggraini Alam ${ }^{3}$ \\ ${ }^{1}$ Faculty of Medicine Universitas Padjadjaran, ${ }^{2}$ Department of Physical Medicine and \\ Rehabilitation Faculty of Medicine Universitas Padjadjaran/Dr. Hasan Sadikin General Hospital, \\ Bandung, ${ }^{3}$ Department of Child Health Faculty of Medicine Universitas Padjadjaran/Dr. Hasan \\ Sadikin General Hospital Bandung
}

\begin{abstract}
Background: Cerebral Palsy (CP) is a group of movement and posture disorder commonly accompanied by comorbidities such as sensation, cognition, communication abnormalities and many more. This study aimed to identify the correlation between gross motor function(measured by Gross Motor Function Classification System, GMFCS) and communication function (measured by Communication Function Classification System, CFCS) in children with CP.

Methods: Thirty six children with CP aged 0-12 years were examined. Samples were taken from Department of Physical Medicine and Rehabilitation Dr. Hasan Sadikin General Hospital Bandung on September to October 2015. Patients' descriptive data, levels of GMFCS and CFCS were collected by the researcher and residents previously standardized. Kendall's tau b correlation coefficient was used to analyze the interrelationship between the GMFCS and CFCS.

Results: Levels of GMFCS and CFCS in all samples were moderately correlated $(r=0.405 ; p=0.004)$. In patients with spastic quadripledic type, correlation were found moderate $(r=0.495 ; p=0.014)$. No significant correlation was found when CP spastic quadriplegic patients were excluded ( $r=0.048, p=0.829)$.

Conclusions: : Levels of GMFCS and CFCS should be described to provide the complete gross motor and communication picture of CP children.Gross motor function in a child with spastic quadriplegic CP might be correctly predicted from his/ her communication function and vice versa.
\end{abstract}

Keywords: Cerebral palsy, children, Communication Function Classification System, Gross Motor Function Classification System

\section{Introduction}

Cerebral palsy (CP) is a group of disorders of movement and posture causing activity limitation that can be attributed to a nonprogressive lesion on developing brain. The disorder is often accompanied by sensation, cognition, communication, perception, behavior abnormalities, and/or episode(s) of seizure. ${ }^{1}$ In 2013, 2.11 out of 1000 children worldwide were born with $\mathrm{CP}^{2}$ Some of the disorders can be classified based on World Health Organization (WHO) International Classification of Functioning, Disability and Health (ICF), which categorizes CP patients on the level of participation. Four assessment tools are proposed; Gross Motor Function Classification System (GMFCS), Communication Function Classification System (CFCS), Manual Ability Classification System (MACS), and Eating and Drinking Ability Classification System (EDACS). ${ }^{3}$ In this study, GMFCS and CFCS were investigated further.

The GMFCS is a method of classification grading functional limitation, use of assitive device, and quality of movement in CP patients. ${ }^{4}$ This classification method has been commonly used, including in Dr. Hasan Sadikin General Hospital Bandung, West Java, Indonesia. Meanwhile, CFCS attempts to classify the ability of a CP patient to communicate consistent messages to other individuals. ${ }^{3}$

Correspondence: Vindy Margaretha Miguna, Faculty of Medicine, Universitas Padjadjaran, Jalan Raya BandungSumedang Km.21, Jatinangor, Sumedang, Indonesia, Phone: +6281321198977 Email: vindymiguna@gmail.com 
Developed in 2011, CFCS can be considered as a new assesment tool. It measures 3 aspects; the ability to alternate being a sender and a receiver, pace of communication, and familiarity of partner.

The correlation becomes important due to the concurrence of gross motor and communication disorder. ${ }^{1}$ Lesion causing CP can occur in various areas causing different gross motor presentation, sometimes even causing dysarthria. ${ }^{3}$ Lesions in the visual cortex, auditory cortex, and neuronal connections will manifest as lingual, both receptive and expressive, and cognitive disorders. All of these would potentially damage the gross motor and communication functions of a person.

From clinical point of view, the correlation identified in this study will provide guidance for professionals to conclude whether or not it is possible to predict a patient's CFCS level based on his/her GMFCS level and vice versa. Previous studies had addressed the issue and findings were variable. In some studies, significant moderate to high correlation was found, another study presented nonsignificant correlation. ${ }^{3,5-7}$ Thus, the aim of this study was to discover the correlation between GMFCS and CFCS with sample taken from Dr. Hasan Sadikin General Hospital (RSHS) Bandung, a tertiary health care facility with a high number of children with $\mathrm{CP}$

\section{Methods}

This study was a cross-sectional study analyzing correlation with categoric ordinal scale of measurement. The subject of this study were children aged $0-12$ years with CP in Department of Physical Medicine and Rehabilitation, Faculty of Medicine, Universitas Padjadjaran, RSHS Bandung. Inclusion criteria were children with $\mathrm{CP}$ aged $0-12$ visiting for their first examination, control, and/or therapy. Any children whose parents refused to give consent were excluded. Minimum sample was 36 calculated by the formula:

$$
n=\left\{\frac{(Z \alpha+Z \beta)}{0,5 \ln \left[\frac{1+r}{1-r}\right]}\right\}^{2}+3
$$

Explanation of formula: $\mathrm{n}$ is the number of sample needed; type 1 error is $5 \%$ and the study was two-sided, $Z \alpha=1.96$; type 2 error is $20 \%, \mathrm{Z} \beta=0.842$; r: minimum valid correlation, determined $\mathrm{r}=0.47$ from previous study with 222 CP patients.

Every CP patient was examined by residents for GMFCS level, and the result was recorded by the researcher. Due to unfamiliarity of researcher to patients' communication function and the unavailability of Indonesian version of CFCS, CFCS measurement were obtained per anamnesis to parents. Anamnesis were done by researcher and residents previously standardized by Physical Medicine and Rehabilitation specialist. Besides GMFCS and CFCS, data recorded as descriptive data of the patients were age group $(0-<2$ years, $2-<4$ years, $4-<6$ years, $6-<12$ years), motoric manisfestation (spastic, ataxic, dyskinetic, hypotonic, mixed type), topography of CP (hemiplegic, diplegic, quadriplegic, triplegic, monoplegic, double hemiplegic), and comorbidities (visual, audio, speech, cognitive disturbances, and seizure episode(s)). Consecutive sampling was used and data were taken during patients' visit to hospital.

This study was conducted in Department of Physical Medicine and Rehabilitation, Faculty of Medicine, Universitas Padjadjaran/ Dr. Hasan Sadikin General Hospital Bandung from September to October 2015. Assesment of GMFCS was conducted according to the classification developed by Palisano et al. ${ }^{4}$, while CFCS was according to study by Hidecker et al. $^{8}$ Correlation between GMFCS and CFCS was analyzed by Kendall's taub correlation. Ethical clearance was approved by The Health Research Ethics Committee, Dr. Hasan Sadikin General Hospital Bandung. Before beginning the observation and anamnesis, informed consent were obtained from parents after receiving explanation regarding what would be done.

\section{Results}

From $36 \mathrm{CP}$ patients, the largest group of patients were spastic quadriplegic aged $2-<4$ years old (Table 1). Almost all patients experienced comorbidities, with speech and cognitive disturbances being the most common. Three patients were without no comorbidities, 5 with 1 comorbidity, 6 with 2 comorbidities, 11 with 3 comorbidities, 7 with 4 comorbidities, and finally 4 with 5 comorbidities.

Functional assessment of GMFCS and CFCS discovered that patients with equal levels for GMFCS and CFCS were 1 spastic hemiplegic (level II), 1 spastic monoplegic (level III), 1 spastic diplegic (level V), and 12 spastic 
Table 1 Characteristics of Children with Cerebral Palsy

\begin{tabular}{lc}
\hline Category & Number of Children \\
\hline Sex & 19 \\
Male & 17 \\
Female & \\
Age Group & 7 \\
0-<2 years & 12 \\
2-<4 years & 11 \\
4-<6 years & 6 \\
6-<12 years & \\
Motoric manifestation & 32 \\
Spastic & 0 \\
Ataxic & 1 \\
Dyskinetic & 2 \\
Hypotonic & 0 \\
Mixed type & 1 \\
Unspecified & \\
Topography & 4 \\
Hemiplegic & 15 \\
Diplegic & 6 \\
Quadriplegic & 21 \\
Triplegic & 0 \\
Monoplegic & 1 \\
Double hemiplegic & 0 \\
Comorbidity & \\
Hearing impairment & \\
Visual impairment & \\
Speech impairment & \\
Cognitive impairment & \\
Seizure episode(s) & \\
\hline & \\
\hline
\end{tabular}

quadriplegic ( 2 patients with level IV, 10 patients with level V) (Figure 1).

Description of scores stratified by type and topography of CP was shown in Figure 2, 3, and 4. Level of GMFCS and CFCS were observed to contain some different combinations.

Some other types of CP samples were 1 spastic monoplegic CP patient with GMFCS level III CFCS level III, 1 dyskinetic CP patient with GMFCS level V CFCS level III, 2 hypotonic CP patients, 1 with GMFCS level IV CFCS level I, 1 with GMFCS level III CFCS level I, and 1 unspecified CP patient with GMFCS level III, CFCS level V.

Correlation between GMFCS and CFCS analyzed by Kendall's tau b was moderate positive and significant $(\mathrm{r}=0.405 ; \mathrm{p}=0.004)$. Stratified by type, there were also a moderate and significant correlation in CP spastic quadriplegic $(\mathrm{r}=0.495 ; \mathrm{p}=0.014)$. In addition to the result, an insignificant correlation $(r=0.048, p=0.829)$ of all other types without CP spastic quadriplegic were present.

\section{Discussions}

This study discovered a moderately positive correlation between GMFCS and CFCS. Thus, a good performance in gross motor may not significantly indicates good communication function and vice versa. This was also the case if finding was a poor function. Result of moderately positive correlation agrees with studies carried out by van der Zwart $^{7}$ and Hidecker et al. ${ }^{6}$ Although mean age was lower, CP type distribution was higher in spastic quadriplegia, and no exclusion criteria

\begin{tabular}{c|ccccc}
\hline GMFCS* $^{*}$ & I & II & III & IV & V \\
\hline I & & 1 & 1 & 1 & 1 \\
II & & 1 & & & \\
III & 2 & 3 & 1 & & 2 \\
IV & 1 & & 4 & 2 & 1 \\
V & 1 & & 2 & 1 & 11 \\
\hline
\end{tabular}

Note: *GMFCS: Gross Motor Function Classification System, ${ }^{* *}$ CFCS: Communication Function Classification System

Figure 1 Distribution of Gross Motor Function Classification System and Communication Function Classification System Levels among Cerebral Palsy Sample 


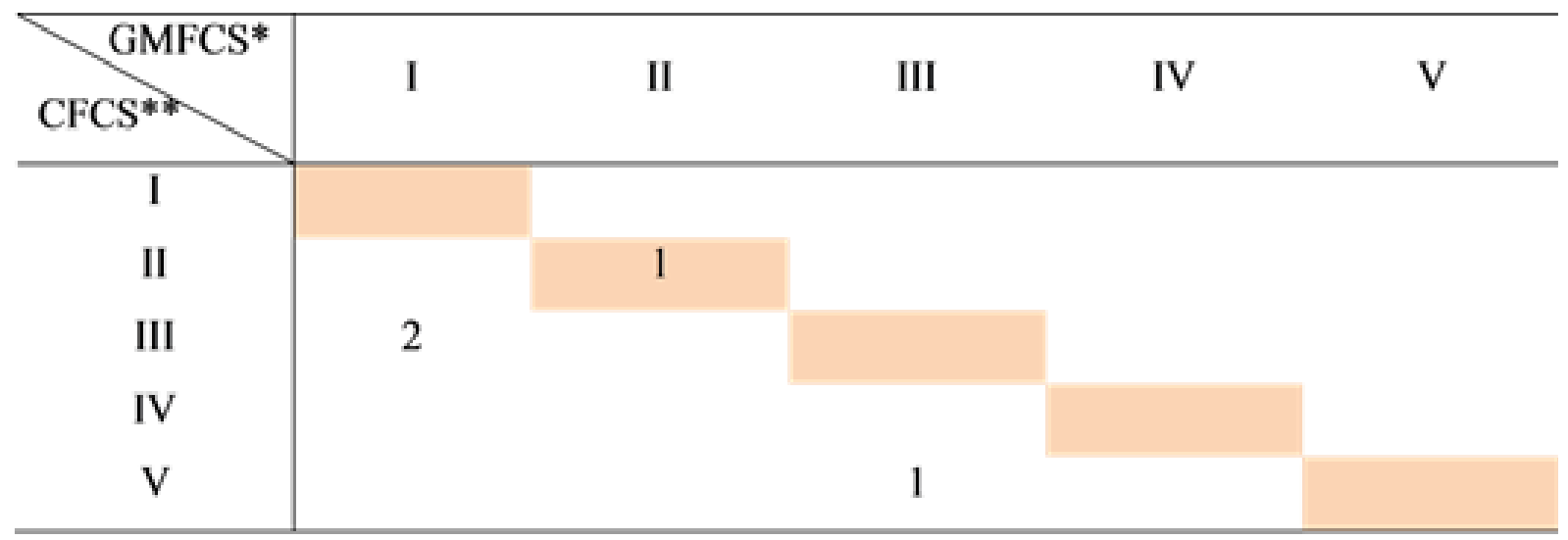

Note: *GMFCS: Gross Motor Function Classification System,**CFCS: Communication Function Classification System

Figure 2 Distribution of Gross Motor Function Classification System and Communication Function Classification System Levels among Spastic Hemiplegic Cerebral Palsy Samples

was proposed in this present study. A partial similarity was found between this study and a study by Compagnone et al. ${ }^{5}$, which analyzed a slightly stronger correlation between GMFCS and CFCS. Possible explanation would be hypoxic-ischemic encepalopathy occuring in extremely diverse region causing distinct presentation or the heterogenous ability of children to cope with their limitations causing different participation function; all explanation results in largely variable findings. ${ }^{9}$

Significant correlation as a conclusion of this study was contrary to a study by Killian et al. ${ }^{3}$ of 49 children with CP recruited from special school for children with complex disability. The past study showed no significant correlation between GMFCS and CFCS. $^{3}$ Disrepancy between results might be due to different settings of study.

Out of 36 samples, 15 had the same levels on GMFCS and CFCS, 11 of whom were in level $\mathrm{V}$, indicating that $\mathrm{CP}$ children with less ability classified in GMFCS were the ones more likely to have the same level of CFCS and vice versa.This finding was different from the observation in studies by Hidecker et al. ${ }^{6}$ and

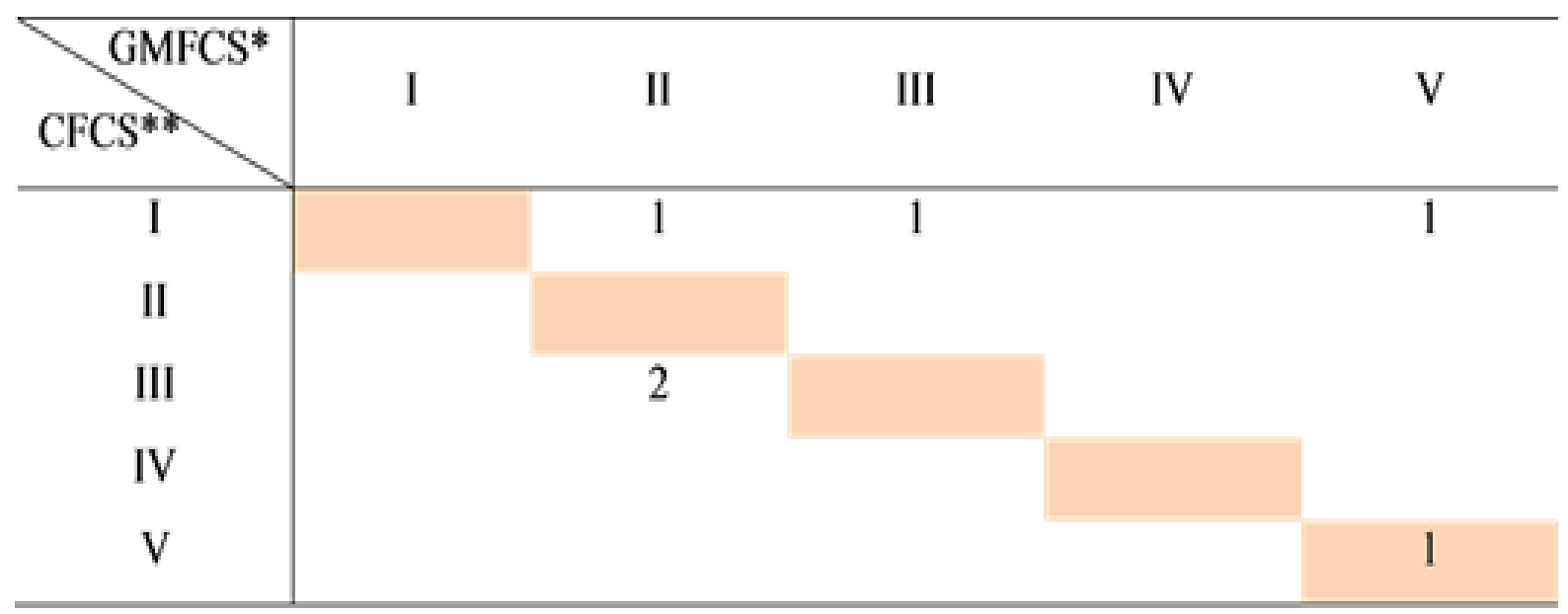

Note: *GMFCS: Gross Motor Function Classification System, ${ }^{* *}$ CFCS: Communication Function Classification System

Figure 3 Distribution of Gross Motor Function Classification System and Communication Function Classification System Levels among SpasticDiplegic Cerebral Palsy Samples 


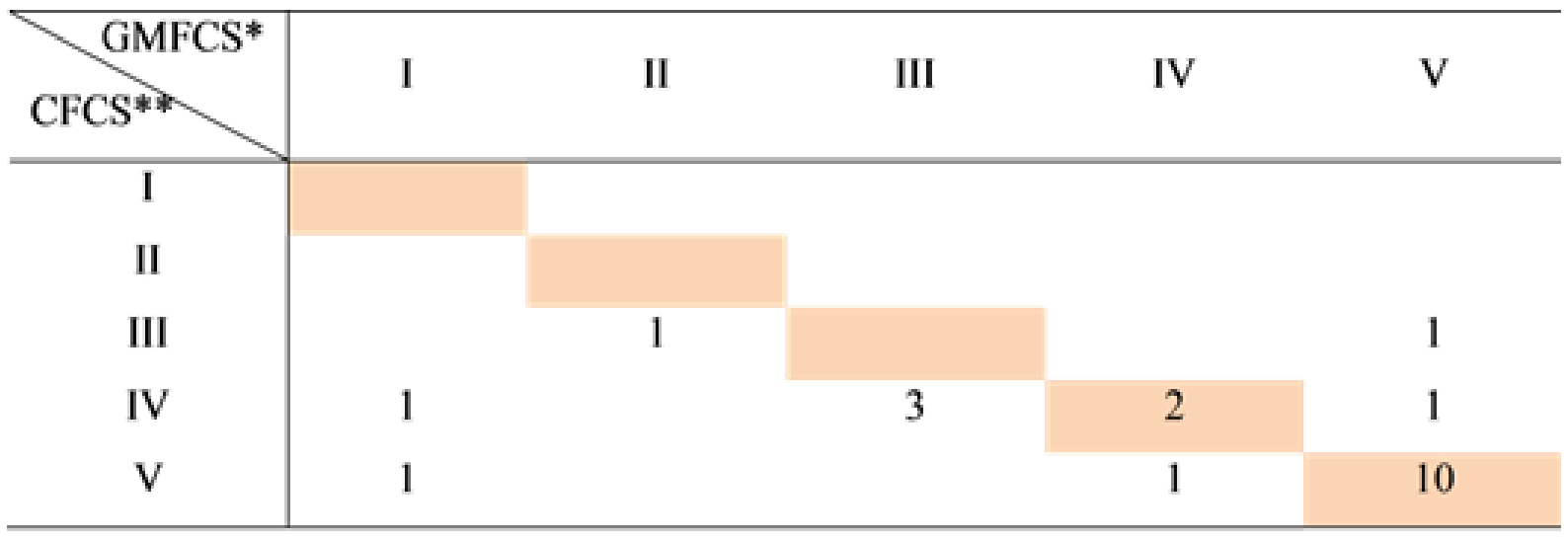

Note: *GMFCS: Gross Motor Function Classification System, ${ }^{* *}$ CFCS: Communication Function Classification System

\section{Figure 4 Distribution of Gross Motor Function Classification System and Communication Function Classification System Levels among SpasticQuadriplegic Cerebral Palsy Samples}

van der Zwart ${ }^{7}$ which found same levels to be most prevalent in level I. The explanation for this was that patients who came to a tertiary health care provider would most probably be of poorer function than clinics and rehabilitation centers as in previous studies.

Several types of CP were observed in this study: spastic quadriplegic, spastic diplegic, spastic hemiplegic, dyskinetic, hypotonic, dan ataxic. Spastic quadriplegic CP was discovered the most (21 out of 36 samples). In this type, the correlation was discovered moderately positive and slightly stronger than when all types were included. Hidecker et al. ${ }^{6}$ on the contrary, revealed that spastic quadriplegic $\mathrm{CP}$ had lower correlation.

Explanation for the distinction can be gained through exploration of sample data distribution in this study. Ten out of 21 patients with spastic quadriplegic CP were classified as GMFCS level V (transported in a manual wheelchair) and CFCS level V (seldom effectively sends and receives, even with familiar partners). These ten were the ones perfectly correlated, creating higherthan-all-types correlation from the previously nonsignificant negatively correlated data without the ten. The reason that almost half of spastic quadriplegic children had such poor gross motor function were clear; spasticity of the entire limbs would impede sitting and walking..$^{10}$ Communication in this study were also of poor due to: 1) Cognitive impairment were present in all spastic quadriplegic sampe. 2) Most spastic quadriplegic children fell into younger children category than the previous study. Communication typically improves as children grew in age. ${ }^{11}$

Interestingly, it was discovered that the correlation of GMFCS and CFCS; if CP spastic quadriplegic type was excluded, then the finding was insignificant. Pathophysiologically speaking, such strong correlation in spastic quadriplegic might be due to severe spasticity in spastic quadriplegic type causing hypoxia leading to worsening of brain functioning. In addition, spasticity occuring not only on the muscles of limbs, but also on the oromotor muscles causes poorer communication function.

Correlation in each type of $\mathrm{CP}$ besides spastic quadriplegic cannot be determined due to inadequacy of samples belonging to those particular types. Nevertheless, the distribution of levels in each type is important to be reviewed. In spastic hemiplegic $\mathrm{CP}$, GMFCS levels were distributed in the "highly functioning" group of level I, II, and III. This fact was in accordance to a study by Himmelman et al. ${ }^{12}$ stating that hemiplegic patients most likely acquire good motoric function.In spastic diplegic type, the finding was also similar. Motoric function were good but observed slightly poorer than hemiplegic patients.This was also noticed on previous research by Shevell et al. ${ }^{10}$ On the contrary, communication were unpredictable in both types, probably owing to the diverse comorbidities suffered as stated in previous studies. ${ }^{13-15}$ For the remainder $\mathrm{CP}$ types, number of samples were limited to one or two. Findings of GMFCS and CFCS levels were not able to be generalized.

Independence of GMFCS and CFCS level in a patient suggested that complete assesment 
of both levels in an individual with $\mathrm{CP}$ will certainly allow better functional potrayal. Besides descriptive purpose, the assessment is also meaningful for patients' therapy. In order to achieve better results, it is imperative that an active participant is practicing meaningful skills for him/her. Hence, the use of functional classifications focusing on participation component such as GMFCS and CFCS as goal setters for therapy is excellent. ${ }^{16}$

The use of functional classification systems in the field of study is unavoidable. Assessment of GMFCS had been used abundantly as an indicator of gross motor ability and CFCS has become increasingly important since it was validated in 2011. Studies had used CFCS to select participant for their study, for predicting communication function, and many more..$^{17,18}$ These evidences provide encouragement for worldwide assesment of GMFCS and CFCS in every CP patients, including in Dr. Hasan Sadikin General Hospital.

Limitation of the study were: 1) hospital setting of the study might have created selection bias. As Dr. Hasan Sadikin General Hospital is a tertiary health care service, patients referred to the hospital consists of mostly "lowfunctioning group". This caused less variability in GMFCS and CFCS combination 2) there was no validated Indonesian version of CFCS yet. To overcome this obstacle, researcher applied interview to parents, which may cause information bias. The weakness of parent reported CFCS was bias of the result towards better function. ${ }^{11} 3$ ) there was no lower limit for age, while CFCS assesment had only been performed in children as young as 2 years. ${ }^{11}$ This study performed the assesment on children as young as 1 year old, which may lead children to be seen as having less ability in communication 4) therapy was not considered in this study, therefore, result might not potray pure correlation.

In conclusion, child's functional capacity in gross motor may not be correctly predicted by his/ her communication function and vice versa, as the correlation of GMFCS and CFCS was moderate. The higher correlation in CP spastic quadriplegic compared to other types implies probable correct prediction of CFCS by assessing level of GMFCS and vice versa in this particular type. Both assesment of GMFCS and CFCS are proven to be a crucial matter in CP children description.

Since the use of GMFCS and CFCS as concurrent assessment of $\mathrm{CP}$ patients are of great importance in the fields of clinical communication, therapy, and research, further studies should also be directed towards the validation of Indonesian-CFCS in order to make it readily available in the country. Cohort studies can also be done to discover whether or not age and therapy have any effect on CFCS level in the long run and its possible correlation with GMFCS.

\section{References}

1. Rosenbaum $P$, Paneth $N$, Leviton $A$, Goldstein M, Bax M, Damiano D, et al. A report: the definition and classification of cerebral palsy April 2006. Dev Med Child Neurol Suppl. 2007;109(Suppl 109):S814.

2. Oskoui M, Coutinho F, Dykeman J, Jette N, Pringsheim T. An update on the prevalence of cerebral palsy: a systematic review and meta-analysis. Dev Med Child Neurol. 2013;55(6):509-19.

3. Killian L, Bryant E, Sellers D. The clinical use of functional classification systems for children and young people with cerebral palsy. Dev Med Child Neurol. 2014;56(Suppls4):32.

4. Palisano R, Rosenbaum P, Walter S, Russell D, Wood E, Galuppi B. Development and reliability of a system to classify gross motor function in children with cerebral palsy. Dev Med Child Neurol. 1997;39(4):214-23.

5. Compagnone E, Maniglio J, Camposeo S, Vespino T, Losito L, De Rinaldis M, et al. Functional classifications for cerebral palsy: correlations between the gross motor function classification system (GMFCS), the manual ability classification system (MACS) and the communication function classification system (CFCS). Res Div Disabil. 2014;35(11):2651-7.

6. Hidecker MJC, Ho NT, Dodge N, Hurvitz EA, Slaughter J, Workinger MS, et al. Inter-relationships of functional status in cerebral palsy: analyzing gross motor function, manual ability, and communication function classification systems in children. Dev Med Child Neurol. 2012;54(8):737-42.

7. van der Zwart K. Communication performance of children with cerebral palsy: relation with spoken language comprehensionand contextual factors [thesis]. Netherlands: Universiteit Utrecht; 2012.

8. Hidecker MJC, Paneth N, Rosenbaum $\mathrm{P}$, Kent RD, Lillie J, Eulenberg JB, et al. Communication Function Classification 
System 2011. [cited 2015 March 31]. Available from: http://cfcs.us/.

9. Berker N, Yalçin S, Root L, Staheli L. The HELP guide to cerebral palsy. 2nded. Seattle: Global HELP Organization; 2010.

10. Shevell MI, Dagenais L, Hall N, The Repacq C. The relationship of cerebral palsy subtype and functional motor impairment: a population-based study. Dev Med Child Neurol. 2009;51(11):872-7.

11. Hidecker MJC, Paneth N, Rosenbaum PL, Kent RD, Lillie J, Eulenberg JB, et al. Developing and validating the communication function classification system for individuals with cerebral palsy. Dev Med Child Neurol. 2011;53(8):70410.

12. Himmelmann $\mathrm{K}$, Beckung $\mathrm{E}$, Hagberg G, Uvebrant P. Gross and fine motor function and accompanying impairments in cerebral palsy. Dev Med Child Neurol. 2006;48(6):417-23.

13. Parkes J, Hill NAN, Platt MJ, Donnelly C. Oromotor dysfunction and communication impairments in children with cerebral palsy: a register study. Dev Med Child Neurol. 2010;52(12):1113-9.

14. Ghasia F, Brunstrom J, Gordon M, Tychsen L. Frequency and severity of visual sensory and motor deficits in children with cerebral palsy: gross motor function classification scale. Invest0phthalmol VisSci. 2008;49(2):572-80.

15. Pruitt DW, Tsai T. Common medical comorbidities associated with cerebral palsy. Phys Med Rehabil Clin N Am. 2009;20(3):453-67.

16. Mayston Am M. From 'one size fits all' to tailor-made physical intervention for cerebral palsy. Dev Med Child Neurol. 2011;53(11):969-70.

17. Choi Y, Lee SH, Chung CY, Park MS, Lee $\mathrm{KM}$, Sung KH, et al. Anterior knee pain in patients with cerebral palsy. Clin Orthop Surg. 2014;6(4):426-31.

18. Coleman A, Weir K, Ware RS, Boyd R. Predicting functional communication ability in children with cerebral palsy at school entry. Dev Med Child Neurol. 2015;57(3):279-85. 